\title{
A Nonparametric Test of Stationarity for Independent Data
}

\author{
Jeffrey D. Hart \\ Texas A\&M University, Department of Statistics
}

\begin{abstract}
A nonparametric test for stationarity of independent data is investigated. The test is based on comparing kernel density estimates calculated from subsamples of the data. Asymptotic distribution theory is developed and results of a modest simulation study are presented.
\end{abstract}

Key words. Randomness statistics; Consistent test; Local alternatives. 


\section{Introduction}

A common problem in statistics is trying to determine whether or not data have a common distribution. This problem arises in quality control, for example, where one wishes to be able to detect some change in a process. Suppose one observes independent random variables $X_{1}, \ldots, X_{N}$ and wants to test whether or not they are stationary, i.e., whether or not they have a common distribution. A num-

ber of methods exist for doing so. One class of methods falls under the heading "change-point detection," which seek to identify an abrupt change in a sequence of observations. The cusum test proposed by Page (1954) is a long-standing tool for detecting change-points. It is designed mainly for detecting level changes in the observed process. Methods for detecting more general types of change, such as in variation or skewness, have also been proposed. These include so-called randomness statistics (McDonald, 1991) and the stationarity tests of Kapetanios (2007), Busetti and Harvey (2010) and Lima and Neri (2013).

The test of stationarity proposed in this paper is an adaptation of the test of Zhan and Hart (2014). Their test is for a setting where one has a large number of small data sets and wishes to test whether all these data sets come from the same distribution. In the current setting, one may partition the $N$ observations into, say, $p$ smaller data sets of equal size, and then apply the test of Zhan and Hart (2014) to these $p$ sets. The statistic of Zhan and Hart (2014) is analogous to one proposed by Lehmann (1951). Let $F^{i}$ be the empirical distribution function (edf) for the $i$ th small data set, $i=1, \ldots, p$, and let $F_{N}$ be the edf for all $N$ observations. Then the statistic of Lehmann (1951) is

$$
\sum_{i=1}^{p} \int\left(F^{i}(x)-F_{N}(x)\right)^{2} d F_{N}(x) .
$$


Lehmann (1951) and McDonald (1991) obtain the asymptotic distribution of (1) in the respective cases (a) $N / p$ tends to $\infty$ with $p$ fixed, and (b) $p$ tends to $\infty$ with $N / p$ fixed.

The statistic of Zhan and Hart (2014) is an analog of (1) that compares kernel density estimates rather than edfs. A number of authors, including Eubank et al. (1994), Martínez-Camblor and de Uña Álvarez (2009) and Rayner et al. (2009), have made the case that goodness-of-fit tests based on density estimates are generally more powerful than ones based on edfs. The simulation study in Section 4 (of the current paper) suggests that a similar conclusion may be valid when testing for stationarity.

The rest of the paper may be outlined as follows. The test statistic and its asymptotic distribution are described in Section 2. Power properties against both fixed and local alternatives are developed in Section 3. A brief simulation study illustrating the potential power gain over edf-based statistics is presented in Section 4. Finally, concluding remarks and proofs of theoretical results are given in Sections 5 and 6 , respectively.

\section{The test statistic and its null distribution}

Let $\boldsymbol{X}=\left(X_{1}, \ldots, X_{N}\right)$ be independent observations such that $X_{i}$ has density $f_{i}$, $i=1, \ldots, N$. We wish to test the null hypothesis

$$
H_{0}: f_{1} \equiv f_{2} \equiv \cdots \equiv f_{N}
$$

against the negation of $H_{0}$. Merely for the sake of notational simplicity, suppose that $n p=N$ for integers $n>1$ and $p$. We assume that $n$ is fixed. Now divide the 
data set into $p$ groups, the $i$ th of which is

$$
\boldsymbol{X}_{i}=\left(X_{n(i-1)+1}, \ldots, X_{n i}\right), \quad i=1, \ldots, p
$$

Having grouped the data in this way, we may now test $H_{0}$ using methodology like that proposed in Zhan and Hart (2014).

The test of Zhan and Hart (2014) is, effectively, a comparison of the $p$ data distributions defined by the above grouping. In a completely arbitrary setting where the alternative hypothesis is true, such a comparison would not necessarily be very powerful. However, the main application envisioned here is that the data are observed chronologically, and changes in densities are such that the difference between $f_{i}$ and $f_{j}$ tends to be larger for large values of $|i-j|$ than for smaller values of $|i-j|$.

For a data set $\boldsymbol{Y}=\left(Y_{1}, \ldots, Y_{k}\right)$, define the following kernel density estimate:

$$
\hat{f}_{h}(x \mid \boldsymbol{Y})=\frac{1}{k b} \sum_{i=1}^{k} \phi\left(\frac{x-Y_{i}}{b}\right),
$$

where $\phi$ is the standard normal density and $b$ is a positive bandwidth. Then our test statistic is a properly standardized version of

$$
\sum_{i=1}^{p} \int_{-\infty}^{\infty}\left(\hat{f}_{b}\left(x \mid \boldsymbol{X}_{i}\right)-\hat{f}_{b}(x \mid \boldsymbol{X})\right)^{2} d x
$$

To define the standardization, let

$$
\begin{aligned}
S_{W} & =\frac{1}{p n(n-1) \sqrt{2} b} \sum_{i=1}^{p} \sum_{j=1}^{n} \sum_{l=1, l \neq j}^{n} \phi\left(\frac{X_{n(i-1)+j}-X_{n(i-1)+l}}{\sqrt{2} b}\right), \\
S_{B} & =\frac{1}{p(p-1) n^{2} \sqrt{2} b} \sum_{i=1}^{p} \sum_{k=1, k \neq i}^{p} \sum_{j=1}^{n} \sum_{l=1}^{n} \phi\left(\frac{X_{n(i-1)+j}-X_{n(k-1)+l}}{\sqrt{2} b}\right) .
\end{aligned}
$$


The proposed test statistic is

$$
T_{b}=\frac{\sqrt{p}\left(S_{W}-S_{B}\right)}{\hat{\sigma}},
$$

where $\hat{\sigma}^{2} / p$ is an estimator (to be defined subsequently) of $\operatorname{Var}\left(S_{W}-S_{B}\right)$ assuming that $H_{0}$ is true.

The reader is referred to Zhan and Hart (2014) for an explanation of why $S_{W}-S_{B}$ is a centered version of (3). When $H_{0}$ is true, $E\left(S_{W}-S_{B}\right)=0$, and under the "smooth" alternatives defined in Section $3 E\left(S_{W}-S_{B}\right)>0$. Furthermore, under hypothesis (2) and with $n$ and $b$ fixed, the results of Zhan and Hart (2014) imply that $T_{b}$ converges in distribution to a standard normal random variable as $N \rightarrow \infty$. The $W$ and $B$ on $S_{W}$ and $S_{B}$ stand for within and between, reflecting the fact that in $S_{W}$ and $S_{B}$ the argument of $\phi$ depends upon two observations that come from the same and different groups, respectively. Our test rejects $H_{0}$ at level $\alpha$ if and only if $T_{b} \geq z_{\alpha}$, where $z_{\alpha}$ is the $1-\alpha$ percentile of the standard normal distribution.

To define the variance estimator $\hat{\sigma}^{2}$, let

$$
\begin{gathered}
h_{1}\left(\boldsymbol{X}_{i}\right)=\frac{1}{n(n-1) \sqrt{2} b} \sum_{j=1}^{n} \sum_{l=1, l \neq j}^{n} \phi\left(\frac{X_{n(i-1)+j}-X_{n(i-1)+l}}{\sqrt{2} b}\right), \\
h_{2}\left(\boldsymbol{X}_{i}, \boldsymbol{X}_{k}\right)=\frac{1}{n^{2} \sqrt{2} b} \sum_{j=1}^{n} \sum_{l=1}^{n} \phi\left(\frac{X_{n(i-1)+j}-X_{n(k-1)+l}}{\sqrt{2} b}\right)
\end{gathered}
$$

and

$$
h_{3}\left(\boldsymbol{X}_{i}\right)=\frac{1}{p-1} \sum_{k=1, k \neq i}^{p} h_{2}\left(\boldsymbol{X}_{k}, \boldsymbol{X}_{i}\right) .
$$

The estimator $\hat{\sigma}^{2}$ is the sample variance of $h_{1}\left(\boldsymbol{X}_{i}\right)-2 h_{3}\left(\boldsymbol{X}_{i}\right), i=1, \ldots, p$, i.e.,

$$
\hat{\sigma}^{2}=\frac{1}{(p-1)} \sum_{i=1}^{p}\left[h_{1}\left(\boldsymbol{X}_{i}\right)-2 h_{3}\left(\boldsymbol{X}_{i}\right)-\hat{\mu}\right]^{2},
$$


where $\hat{\mu}=\sum_{i=1}^{p}\left[h_{1}\left(\boldsymbol{X}_{i}\right)-2 h_{3}\left(\boldsymbol{X}_{i}\right)\right] / p$. Zhan and Hart (2014) show that, when $H_{0}$ is true and $p \rightarrow \infty, \hat{\sigma}^{2}$ consistently estimates $\lim _{p \rightarrow \infty} p \operatorname{Var}\left(S_{W}-S_{B}\right)$.

In practice one must choose a value for the bandwidth $b$. Simulation studies have verified that the bandwidth can have an important effect on power of the test. To deal with this effect, we propose that one compute the test statistic at several different bandwidths, and then reject $H_{0}$ when the largest of these statistics exceeds an appropriately adjusted critical value. A conservative adjustment can be obtained using the Bonferroni inequality. If one computes test statistics at $m$ different bandwidths, then an asymptotically valid level $\alpha$ test would reject $H_{0}$ when the largest of these statistics exceeds $z_{\alpha / m}$. A less conservative approach was proposed by Zhan and Hart (2014), who suggested that one use simulation to approximate the null distribution of $\max _{b} T_{b}$. This is the approach that will be used in our simulation study.

\section{$3 \quad$ Asymptotic power properties}

Let $B$ be a set of $m$ bandwidths, and define $T=\max _{b \in B} T_{b}$. Determining the precise large sample distribution of $T$ will not be undertaken here, but as previously discussed, an asymptotically valid level $\alpha$ test would reject $H_{0}$ for $T \geq z_{\alpha / m}$. The power of this test is at least as big as $\min _{b \in B} P\left(T_{b} \geq z_{\alpha / m}\right)$, and hence we may obtain valuable information about the power of a test based on $T$ by considering the simpler test statistic $T_{b}$ for a given $b$.

We consider alternatives to $H_{0}$ in which the difference between densities is parameterized. Let $\{f(\cdot \mid \boldsymbol{\theta}): \boldsymbol{\theta} \in \Theta\}$ be an identifiable family of densities with 
continuous $d$-dimensional parameter space $\Theta$. Define alternatives as follows:

$$
f_{i}(x)=f(x \mid \boldsymbol{\theta}(i / N)), \quad i=1, \ldots, N,
$$

where $\boldsymbol{\theta}(t)$ is a $d$-variate function of the scalar $t, 0 \leq t \leq 1$, taking on values in $\Theta$. For example, suppose that $\boldsymbol{\theta}=\left(\theta_{1}, \theta_{2}\right)$ and $f(\cdot \mid \boldsymbol{\theta})$ is a normal density with mean $\theta_{1}$ and variance $\theta_{2}$. If the alternative is such that the mean of the distributions changes over time but the variance remains constant, then we would have $\boldsymbol{\theta}(t)=\left(\theta_{1}(t), \theta_{2}\right)$.

The following lemma provides an asymptotic approximation for $E\left(S_{W}-S_{B}\right)$, the key determinant in the power of our test. Proofs of subsequent results are given in the Appendix.

Lemma 1. Suppose that the underlying densities are of the form (4) with $f(x \mid \boldsymbol{\theta}(t))$ uniformly bounded in $x$ and $t$. Suppose also that, as a function of $t, f(x \mid \boldsymbol{\theta}(t))$ is uniformly Lipschitz continuous in the sense that

$$
|f(x \mid \boldsymbol{\theta}(s))-f(x \mid \boldsymbol{\theta}(t))| \leq D|s-t|
$$

for some constant $D$ and all $x, s$ and $t$. Then

$$
E\left(S_{W}-S_{B}\right)=\mu_{b}+O\left(\frac{1}{p}\right)
$$

where

$$
\mu_{b}=\frac{1}{2} \int_{0}^{1} \int_{0}^{1} \int_{-\infty}^{\infty}\left[f_{b}(x \mid \boldsymbol{\theta}(s))-f_{b}(x \mid \boldsymbol{\theta}(t))\right]^{2} d x d s d t
$$

and

$$
f_{b}(x \mid \boldsymbol{\theta}(t))=\int_{-\infty}^{\infty} \frac{1}{b} \phi\left(\frac{x-y}{b}\right) f(y \mid \boldsymbol{\theta}(t)) d y, \quad \text { for all } x \text { and } t .
$$


Not surprisingly, the numerator of our test statistic estimates a quantity that measures how much the densities $f(\cdot \mid \boldsymbol{\theta}(t))$ vary with respect to $t$. Note that for any fixed $t, f_{b}(\cdot \mid \boldsymbol{\theta}(t))$ is the expected value of a Gaussian kernel density estimate for a random sample from $f(\cdot \mid \boldsymbol{\theta}(t))$. Identifiability of the model and the Lipschitz condition in Lemma 1 guarantee that $\mu_{b}$ is strictly positive so long as $\boldsymbol{\theta}(t)$ is a nonconstant function of $t$.

The following theorem shows that our test is generally consistent for fixed alternatives of the form (4), and can detect local alternatives that converge to the null no faster than rate $p^{-1 / 4}$.

Theorem 1. Let the conditions of Lemma 1 hold. Assuming that $\boldsymbol{\theta}(t)$ is not a constant function of $t$, and letting $C$ be any constant, the power of the test with rejection region $T_{b} \geq C$ tends to 1 as $N \rightarrow \infty$. Now suppose that the underlying densities are mixtures of the form

$$
f_{i}(x)=\left(1-p^{-1 / 4}\right) f(x)+p^{-1 / 4} f(x \mid \boldsymbol{\theta}(i / N)),
$$

where $f(x \mid \boldsymbol{\theta}(t))$ satisfies the conditions of Lemma 1. Then

$$
\lim _{N \rightarrow \infty} P\left(T_{b} \geq C\right)=\Phi\left(C-\frac{\mu_{b}}{\sigma}\right)
$$

where $\Phi$ is the standard normal cumulative distribution, $\mu_{b}$ is as defined in Lemma 1 and $\sigma^{2}$ is the limit of $p \operatorname{Var}\left(S_{W}-S_{B}\right)$ assuming that $H_{0}$ is true.

To this point we have not addressed the choice of $n$. Ideally one would choose $n$ to optimize power. For the smooth alternatives we have considered, the asymptotic value of $E\left(S_{W}-S_{B}\right)$ does not depend on $n$. However, $p \operatorname{Var}\left(S_{W}-S_{B}\right)$ does depend on $n$, even in the limit. For the sake of power, it is obviously better for $\operatorname{Var}\left(S_{W}-S_{B}\right)$ 
to be small, which will be the case for $n$ larger. But increasing $n$ decreases $p$, which also tends to reduce power. So, there is undoubtedly a happy medium, i.e., some optimum choice of $n$. One possibility would be to treat $n$ just like $b$ and choose the two quantities to maximize the value of the test statistic. One would then need to adjust critical values to ensure a desired level for the test.

\section{Simulation study}

Here we present results from a small simulation study. Its purpose is to show the potential gain in power that is possible when using our proposed test rather than the edf statistic (1). We consider four cases where $N=1000$ and $n=10$. The null distribution of (1), which is invariant to location and scale, was approximated by generating 5000 independent random samples of size 1000 from the standard normal distribution. The 95th percentile of this distribution was found to be approximately 0.0190. Defining the set $B=\{0.10,0.20, \ldots, 2\}$, we also considered tests based on $T^{\max }=\max _{b \in B} T_{b}$. Simulation, again based on 5000 replications from the standard normal distribution, was used to approximate the null distribution of $T^{\max }$. Interestingly, the approximate 95 th percentile of $T^{\max }$ turned out to be 1.563 , which is smaller than $z_{.05}=1.645$. This reflects the fact that the distribution of each $T_{b}$ tends to be somewhat skewed to the left for "small" values of $p$ (in this case 100), so a right-tailed test based on $N(0,1)$ is somewhat conservative (Zhan and Hart, 2014).

For each of four different cases we generated 1000 sets of 1000 independent observations. On each data set of size 1000 we performed nominal level 0.05 tests based on (1) and $T^{\max }$, using the percentiles reported immediately above. In the first case considered, each data set was a random sample of size 1000 from $N(0,1)$, 
as a check on the percentiles previously obtained. The other three cases were ones in which the alternative was true. In one case $X_{i} \sim N\left(i / 1000,1.4^{2}\right), i=1, \ldots, 1000$, and hence the mean of the data increases linearly but the standard deviation is fixed. In another case $X_{i} \sim N\left(0,(1+3(i / 1000))^{2}\right), i=1, \ldots, 1000$, which has constant mean and a standard deviation that increases linearly. Finally, we considered a setting where the first 800 data values come from $N(0,1)$ and the last 200 from

$$
f_{a}(x)=w \frac{1}{\sigma} \phi\left(\frac{x-\mu_{1}}{\sigma}\right)+(1-w) \frac{1}{\sigma} \phi\left(\frac{x-\mu_{2}}{\sigma}\right)
$$

where $\left(w, \mu_{1}, \mu_{2}, \sigma\right)=(0.2,-1.940,0.485,0.243)$. The density $f_{a}$ is a bimodal mixture of normals and has mean 0 and variance 1 . The first two moments were chosen to match those of $N(0,1)$ to ensure that when a test rejected $H_{0}$, it was not doing so because of location and/or scale changes.

The particular parameters of each alternative were chosen so that the edf test had power of approximately 0.70 . Results are summarized in Table 1 . The power of the kernel-based test is statistically significantly larger (with $\alpha<0.0005$ ) than that of the edf statistic in each of the three alternative cases. Only in the case of a mean change is the power of the kernel-based test not substantially larger. This fits with other results comparing edf statistics and ones based on density estimators. For example, Eubank et al. (1994) point out that the Cramér-von Mises goodness-of-fit statistic (a close cousin of (1)) tends to have relatively good power only against location and scale alternatives. This is because that statistic is a weighted sum of components corresponding to different moments, and the moments of order higher than the variance are greatly downweighted. 
Table 1: Estimated power of EDF and kernel-based tests. Each table value is the proportion of rejections in 1000 independent replications. All tests were performed at nominal significance level 0.05 .

\begin{tabular}{lr|r|r|r} 
& \multicolumn{3}{|c}{ Distribution } \\
\cline { 2 - 5 } Test & $N(0,1)$ & Mean change & Variance change & Normal mixture \\
\hline \hline EDF & 0.040 & 0.689 & 0.684 & 0.723 \\
\hline Kernel-based & 0.045 & 0.722 & 1.000 & 1.000
\end{tabular}

\section{Concluding remarks}

A test of Zhan and Hart (2014) based on kernel density estimators has been adapted to provide a test of stationarity for independent data. The large sample distribution of the test under the null hypothesis of stationarity follows immediately from results of Zhan and Hart (2014). The new test is shown to be consistent for a large class of alternatives to $H_{0}$, each of which consists of densities that change smoothly with the data index. It is also shown that the test can detect local alternatives that converge to the null hypothesis at rate $N^{-1 / 4}$, where $N$ is sample size. A simulation study and results from the literature suggest that the new test is generally at least as powerful as an existing test of stationarity based on empirical distribution functions.

Some aspects of the new test deserve further study. Principal among these is the best way to choose $n$ and the bandwidth $b$. For cases where $N$ is not particularly large it would be worthwhile to study use of the bootstrap to approximate the null distribution of the test statistic. Finally, a comprehensive comparison of power should be conducted for the proposed kernel-based test, edf tests and the stationarity tests of Kapetanios (2007), Busetti and Harvey (2010) and Lima and Neri (2013). 


\section{Appendix}

Proof of Lemma 1. We have

$$
\begin{aligned}
E\left(S_{W}\right)= & \frac{1}{p n(n-1) \sqrt{2} b} \sum_{i=1}^{p} \sum_{j=1}^{n} \sum_{l=1, l \neq j}^{n} \iint \phi\left(\frac{x-y}{\sqrt{2} b}\right) \\
& \times f\left(x \mid \boldsymbol{\theta}\left(\frac{n(i-1)+j}{N}\right)\right) f\left(y \mid \boldsymbol{\theta}\left(\frac{n(i-1)+l}{N}\right)\right) d x d y .
\end{aligned}
$$

Using the smoothness condition of the lemma, it follows that

$$
\begin{aligned}
E\left(S_{W}\right) & =\frac{1}{p \sqrt{2} b} \sum_{i=1}^{p} \iint \phi\left(\frac{x-y}{\sqrt{2} b}\right) f(x \mid \boldsymbol{\theta}(i / p)) f(y \mid \boldsymbol{\theta}(i / p)) d x d y+O\left(\frac{1}{p}\right) \\
& =\frac{1}{\sqrt{2} b} \int_{0}^{1} \iint \phi\left(\frac{x-y}{\sqrt{2} b}\right) f(x \mid \boldsymbol{\theta}(t)) f(y \mid \boldsymbol{\theta}(t)) d x d y d t+O\left(\frac{1}{p}\right) .
\end{aligned}
$$

In exactly the same way we may show that

$$
E\left(S_{B}\right)=\frac{1}{\sqrt{2} b} \int_{0}^{1} \int_{0}^{1} \iint \phi\left(\frac{x-y}{\sqrt{2} b}\right) f(x \mid \boldsymbol{\theta}(s)) f(y \mid \boldsymbol{\theta}(t)) d x d y d s d t+O\left(\frac{1}{p}\right) .
$$

The difference between the integrals in (5) and (6) is

$$
\begin{aligned}
\frac{1}{2 \sqrt{2} b} \int_{0}^{1} \int_{0}^{1} \iint \phi\left(\frac{x-y}{\sqrt{2} b}\right)[f(x \mid \boldsymbol{\theta}(s))-f(x \mid \boldsymbol{\theta}(t))] \\
\times[f(y \mid \boldsymbol{\theta}(s))-f(y \mid \boldsymbol{\theta}(t))] d x d y d s d t
\end{aligned}
$$

Using the fact that

$$
\frac{1}{\sqrt{2} b} \phi\left(\frac{x-y}{\sqrt{2} b}\right)=\int \frac{1}{b} \phi\left(\frac{x-u}{b}\right) \frac{1}{b} \phi\left(\frac{y-u}{b}\right) d u
$$

it is easy to show that integral (7) is $\mu_{b}$, and the result follows.

The proof of Theorem 1 is straightforward using Lemma 1 and essentially the 
same techniques as in Zhan and Hart (2014).

\section{Acknowledgements}

This research was supported in part by National Science Foundation Grant DMS0604801.

\section{References}

Busetti, F. and A. Harvey (2010). Tests of strict stationarity based on quantile indicators. Journal of Time Series Analysis 31, 435-450.

Eubank, R. L., J. D. Hart, and V. N. LaRiccia (1994). Testing goodness of fit via nonparametric function techniques. Communications in Statistics 22, 3327-3354.

Kapetanios, G. (2007). Testing for strict stationarity. Working Paper 602, Department of Economics, University of London.

Lehmann, E. L. (1951). Consistency and unbiasedness of certain nonparametric tests. Annals of Mathematical Statistics 22, 165-179.

Lima, L. and B. Neri (2013). A test for strict stationarity. In V.-N. Huynh, V. Kreinovich, S. Sriboonchitta, and K. Suriya (Eds.), Uncertainty Analysis in Econometrics with Applications, pp. 17-30. Springer-Verlag Berlin Heidelberg.

Martínez-Camblor, P. and J. de Uña Álvarez (2009). Nonparametric $k$-sample tests: Density functions vs. distribution functions. Computational Statistics and Data Analysis 53, 3344-3357.

McDonald, D. (1991). On the asymptotics of randomness statistics. Canadian Journal of Statistics 19, 209-217. 
Page, E. S. (1954). Continuous inspection scheme. Biometrika 41, 100-115.

Rayner, J. C. W., O. Thas, and D. J. Best (2009). Smooth Tests of Goodness of Fit: Using $R$ (2nd ed.). Singapore: Wiley.

Zhan, D. and J. D. Hart (2014). Testing equality of a large number of densities. Biometrika 101, 449-464. 